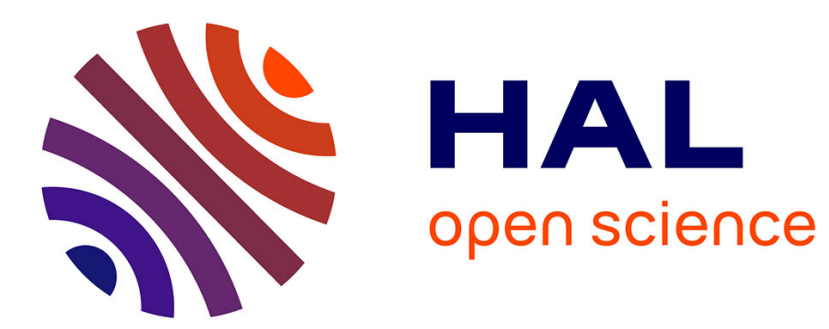

\title{
Les voyageurs de langue française et la domination vénitienne sur le royaume de Chypre (1480-1550)
}

Gilles Grivaud

\section{To cite this version:}

Gilles Grivaud. Les voyageurs de langue française et la domination vénitienne sur le royaume de Chypre (1480-1550). Cahiers du Centre d'Etudes Chypriotes, 2013. hal-01937353

HAL Id: hal-01937353

https://hal-normandie-univ.archives-ouvertes.fr/hal-01937353

Submitted on 28 Nov 2018

HAL is a multi-disciplinary open access archive for the deposit and dissemination of scientific research documents, whether they are published or not. The documents may come from teaching and research institutions in France or abroad, or from public or private research centers.
L'archive ouverte pluridisciplinaire HAL, est destinée au dépôt et à la diffusion de documents scientifiques de niveau recherche, publiés ou non, émanant des établissements d'enseignement et de recherche français ou étrangers, des laboratoires publics ou privés. 


\title{
LES VOYAGEURS DE LANGUE FRANÇAISE et la domination vénitienne sur le royaume de Chypre (1480-1550)
}

\author{
Gilles GRIVAUD
}

\begin{abstract}
Unlike the French travelers of the nineteenth century, those of the years 1480 to 1550 have not considered Cyprus as a country with specific links with the kingdom of France, despite the long period of Lusignan's domination. In their eyes, the island was a country part of the Christian West, even if the indigenous population had specifics ritual practices; testimonies expressing resentment from the Frankish elites against Venice are exceptional.
\end{abstract}

L'histoire culturelle du royaume de Chypre à la fin du Moyen Âge est marquée par un double processus, qui a été clairement observé et formulé, d'abord par Nicolas Iorga au tournant des années 1930, puis, de manière mieux documentée, par Jean Richard ${ }^{1}$. Les deux historiens se sont accordés pour constater, d'une part, un lent affaiblissement de la culture d'expression franque, qui brille de ses plus beaux feux de la fin du XII siècle au milieu du XIV ${ }^{e}$, alors que, d'autre part, la culture de langue grecque et celle d'expression italienne entrent dans une période d'épanouissement durant les $\mathrm{XV}^{\mathrm{e}}$ et $\mathrm{XVI}^{\mathrm{e}}$ siècles ${ }^{2}$.

Les derniers témoins d'une production littéraire originale en langue française appartiennent au règne de Jean II (1432-1458), même si le français reste la langue des actes de l'administration des Lusignan jusqu'à la disparition de Jacques II, le 6 juillet $1473{ }^{3}$. Dans cette perspective, l'année de l'installation du pouvoir vénitien sur l'île (1474), marque une date charnière dans l'histoire des pratiques culturelles insulaires, puisque l'italien remplace définitivement le français comme langue des institutions. Ce basculement majeur, qui résulte d'un enchaînement particulier d'événements politiques et

1. Iorga 1931, p. 194-214 et l'étude de Mureşan supra ; Richard 1987.

2. Ces conclusions ont été reprises et complétées par : Nicolaou-Konnari 1995 ; Grivaud 2009, p. 19-33.

3. Pour les actes de l'administration des Lusignan au Xv siècle : Richard 1962, p. 123-157, et Richard 1983 ; pour les textes littéraires : Grivaud 2001. 
militaires, entérine une évolution engagée depuis la fin du siècle précédent, car les élites aristocratiques du royaume franc s'étaient délibérément tournées vers les universités italiennes pour leur confier la formation intellectuelle de leur progéniture masculine ${ }^{4}$. L'établissement de l'italien comme langue officielle du Regno sanctionnait le cours naturel des échanges diplomatiques et commerciaux, mais aussi le développement des affinités culturelles, qui voyaient triompher l'influence de Venise aux dépens de celle du royaume des Valois.

Les témoignages sur les aspects pris par cette transition culturelle frappent par leur minceur lorsqu'on interroge les sources produites à Chypre après 1474. Les officiers vénitiens ont longtemps déploré l'insuffisance des moyens humains et techniques mis à leur disposition pour exploiter les codes de lois et les registres de fiefs dressés en français, textes qui fondaient les relations entre les représentants de l'autorité coloniale et les classes sociales insulaires ${ }^{5}$. On ignore tout cependant des réactions des membres des maisons aristocratiques d'origine franque ou des bourgeois des villes qui avaient été associés à l'administration des Lusignan. Sans doute peut-on supposer que la mutation linguistique souleva peu de problèmes chez ces groupes, tant les élites étaient depuis longtemps rompues aux pratiques multiculturelles; l'adaptation linguistique semblait fréquente au sein-même des bureaux de l'administration royale puisque le Livre des Remembrances de la Secrète de l'année fiscale 1468 contient des règlements rédigés dans la langue d'usage des destinataires, le français en règle générale, mais aussi l'italien pour les habitants de Famagouste, et le grec pour les higoumènes des monastères de rite orthodoxe ${ }^{6}$. De fait, dès la fondation du royaume franc, la chancellerie des Lusignan alternait l'usage du latin, du français, du grec, voire de l'arabe, en fonction des circonstances ; progressivement, l'emploi de l'italien était venu compléter le répertoire initial ${ }^{7}$. Ce pluralisme linguistique, qui reflète les pratiques des milieux urbains - auxquels on associe les personnels liés aux administrations royales, ecclésiastiques et seigneuriales -, générait, on s'en doute, d'infinies situations d'hybridation linguistique ${ }^{8}$.

Cette habitude des pratiques multiculturelles, dont on ne saurait exagérer l'originalité ou la complexité en milieu méditerranéen et levantin, a diversement retenu l'attention des voyageurs français qui ont visité Chypre. Au cours du XIX ${ }^{\mathrm{e}}$ siècle, nombreux furent ceux qui, emportés par leurs sentiments nationaux-romantiques, exaltèrent le passé mythique des croisades et la domination des Lusignan sur Chypre, sans cacher leur nostalgie d'une époque glorieuse pour la nation contemporaine. Le plus souvent, ils minimisèrent la

4. Référence étant faite à la fondation que Pierre de Cafran institue au bénéfice d'étudiants pour qu'ils se rendent à l'université de Padoue en 1393 : Tselikas 1988-1989 ; Grivaud 2009, p. 40-41.

5. Grivaud 1992, p. 541-546.

6. Richard 1983.

7. Richard 1986, p. 77-83 ; Richard 2003 ; Beihammer 2007.

8 . Sur cet aspect, déjà relevé par Richard, Grivaud et Nicolaou-Konnari ( $c f$. les travaux cités en notes 1 et 2), on retiendra surtout l'étude approfondie de Baglioni 2006. 
dimension multiculturelle de la société insulaire médiévale pour insister sur la force du « génie national » capable d'édifier des cathédrales gothiques et de puissants châteauxforts à machicoulis dans un paysage planté de grenadiers et de palmiers. Ancrés dans les courants idéologiques de leur époque, ces voyageurs considéraient l'île comme un rameau flamboyant de la culture franque médiévale, un rameau se rattachant à la « mèrepatrie » de manière évidente et naturelle ${ }^{9}$.

La construction d'un lien idéalisé entre la métropole - le royaume de France - et sa colonie - le royaume de Chypre - relève d'un fantasme qui correspond évidemment à la formulation des rapports internationaux propres au XIX ${ }^{\mathrm{e}}$ siècle, non à la réalité médiévale. Pour preuve, on examinera le contenu des récits laissés par des voyageurs de langue française ayant visité Chypre dans les décennies qui ont suivi l'installation de la domination vénitienne sur l'île. Cette période est en effet éclairée par un nombre important de textes, produits par des clercs ou des laïcs se rendant en pèlerinage à Jérusalem et en Terre sainte ; tous bénéficient de la sécurité que leur assurent les galées pèlerines affrétées à Venise, une ou deux fois par an, sécurité qui les contraint souvent à une halte de deux semaines à Chypre, au retour de Jaffa en règle générale ${ }^{10}$.

Les voyageurs profitent de l'immobilisation aux Salines pour se rendre à Famagouste ou à Nicosie, dont ils admirent à la fois les enceintes, les maisons et les cathédrales ; si les visiteurs accomplissent à l'occasion l'excursion jusqu'au sommet de la montagne de Stavrovouni, tous observent le port de Limassol et, plus rarement, celui de Paphos, dont ils laissent des descriptions assez détaillées ${ }^{11}$. De cet itinéraire balisé, il ressort que les récits

9. Quelques exemples : «Il vaut mieux, pour l'île de Chypre, s'en tenir à la poésie qu'à l'histoire [...] c'est une singulière chose à se représenter que les temples d'Amathonte et d'Idalie convertis en donjons dans le moyen-âge. Un gentilhomme françois était roi de Paphos, et des barons couverts de leurs hoquetons étoient cantonnés dans les sanctuaires de Cupidon et des Grâces »: Chateaubriand 1836, p. 69. En 1820, lors de sa visite de l'église/mosquée Sainte-Sophie de Nicosie, le comte de Marcellus rapporte : «Je ne pouvais me lasser de me promener d'un pilier à l'autre sous ces longues voûtes gothiques, élancées et sombres, comme nos belles cathédrales françaises : et quand mes yeux retrouvaient l'élégante ogive, ils cherchaient aussi l'autel, l'orgue, enfin la croix, et retombaient avec douleur sur le pupître de l'Imam »: Marcellus 1839, vo. I, p. 317. Logiquement, dix ans plus tard, Joseph Michaud espérait que la mosquée serait un jour rendue au culte chrétien : Michaud, Poujoulat 1833-1835, vol. IV, p. 73. Le comte de Mas-Latrie, pourtant fin expert de la civilisation franque de l'île, défend, de manière tout aussi explicite, le point de vue de l'identité française du royaume : "En France, sur une superficie égale à celle de l'île de Chypre, on trouverait une population d'un demi-million d'habitants. À la fin du règne des rois français, après des guerres et des émigrations désastreuses, on comptait encore en Chypre une population de 147000 habitants » [ ]. «Je suis bien loin de pouvoir rapprocher, avec preuve, de chaque nom géographique grec le véritable nom français lui correspondant du temps de nos princes »: Mas Latrie 1879, p. 159, 162 [c'est nous qui soulignons] ; l'attitude de Mas Latrie est analysée plus en détail infra, dans l'étude d'A. Nicolaou-Konnari.

10. Sur les conditions de l'étape chypriote des pèlerins de Terre sainte : Grivaud 1990, p. 23-25.

11. Au demeurant, on relève que l'itinéraire de ces voyageurs ne diffère pas de ceux des voyageurs de la première moitié du $\mathrm{Xv}^{\mathrm{e}}$ siècle : ibid. 
présentent de nombreuses analogies, reflétant une vulgate colportée par les marins, les taverniers, les caravaniers et d'autres intermédiaires qui, agissant comme d'authentiques guides professionnels, transmettent les informations nécessaires aux pèlerins durant leur escale chypriote. Entre 1480 et le milieu du Xvi siècle, dix-sept témoignages peuvent être soumis à l'enquête pour restituer la perception de Chypre que retinrent des voyageurs de langue et de culture française, voyageurs qui offrent un profil assez homogène d'un point de vue socio-culturel, puisque tous sont issus de milieux urbains, provenant de villes placées soit sous l'autorité des derniers Valois, soit sous celle des ducs de Bourgogne ${ }^{12}$.

En règle générale, le statut politique de l'île ne suscite guère d'attention car, en premier lieu, les pèlerins considèrent Chypre comme un pays chrétien, où sont célébrés à la fois le culte latin et le culte grec ; ultime place fréquentée avant d'atteindre le monde musulman, l'île est le poste-frontière où il convient d'accomplir ses dévotions dans les églises de rite latin. Le passage des pèlerins à Limassol révèle leur attitude inquiète et fervente ; ceux-ci se rassurent à observer que la cathédrale est desservie par quatre ou cinq chanoines qui proviennent, selon les versions, de Picardie, de Bourgogne, de Languedoc, d'Italie ou de Catalogne ${ }^{13}$; la présence de si nombreuses nations familières convainc de l'intégration de l'île à la chrétienté latine, malgré l'éloignement imposé par deux ou trois semaines de navigation ; tous dessinent l'image d'un microcosme où domine le rite latin, appuyé sur une hiérarchie ecclésiastique conforme à celle qui structure l'Occident romain, renforcée par le déploiement des ordres mendiants, puisqu'ils relèvent l'existence d'établissements de franciscains à Limassol ${ }^{14}$, à Nicosie ${ }^{15}$, et à Paphos ${ }^{16}$, de dominicains et de carmes à Nicosie ${ }^{17}$. La fragilité de l'installation latine transparaît cependant, car Sébastien Mamerot note avec précision les injures commises par les Mamelouks sur le mobilier des églises de Limassol, faisant sans doute référence aux dévastations commises entre 1424 et $1426^{18}$.

12. Chronologiquement, il s'agit de : Nicole Le Huen (1479 ?), l'anonyme de 1480, Pierre Barbatre (1480), Sébastien Mamerot (c. 1480), Georges Lengherand (1486), Jean de Cucharmoys (1490), Philippe de Voisins (1490), Pierre Mésenge et Charles de La Rivière (1507), Jacques Le Saige (1518), Greffin Affagart (1519, 1533-1534), Nicolas Loupvant (1531), Denis Possot (1532), l'anonyme de 1546, Pierre Le Boucq (1548) et Antoine Regnaut (1549). Nous avons inclus à cette liste le récit de Barthélémy de Salignac (avant 1525), bien que le bref texte reprenne nombre de topoi de la littérature de voyage et n'apporte aucune nouvelle information ou remarque inédite.

13. Sébastien Mamerot, p. 90 ; anonyme de 1480, p. 107 ; Pierre Barbatre, p. 99 ; Jacques Le Saige, p. 93 ; Denis Possot, p. 149.

14. Pierre Le Boucq, fol. $32 \mathrm{v}^{\circ}$; Denis Possot, p. 149.

15. Charles de La Rivière, fol. 84r ${ }^{\circ}$; Greffin Affagart, p. 244.

16. Nicole Le Huen, fol. n. n.; Pierre Barbatre, p. 100 ; anonyme de 1546, fol. 17r.

17. Nicole Le Huen, p. 90 ; Charles de La Rivière, fol. $84 \mathrm{r}^{\circ}$; Pierre Le Boucq, fol. $32 v^{\circ}$.

18. Sébastien Mamerot, p. 90 : «Tour les ymaiges des eglises sont decoupees, dehachees \& deffigurees desditz Sarrazins qui ont couppe lesdictes ymaiges painctes contre les murs, tant le crucifix, nostre Dame, les evangelistes que autres sainctz ». 
Cette présence déterminante du culte latin dans les villes n'occulte pas le fait que la population insulaire, dans sa majorité, professe le rite grec, tant dans les campagnes que dans les villes. Nos voyageurs remarquent leur cathédrale à Limassol ${ }^{19}$, et surtout le nombre important de leurs lieux de culte à Nicosie, Charles de La Rivière rapportant le chiffre de trente-quatre églises dans la capitale ${ }^{20}$. Dans une église voisine de SainteSophie, dont ils ignorent le vocable (Odigitria ?) et qui est présentée comme une collégiale, Charles de La Rivière constate la dévotion des prêtres officiant ; Nicolas Loupvent en décrit leur costume sans savoir distinguer s'il s'agit de moines ou de prêtres ${ }^{21}$. Pour la plupart des pèlerins, Chypre représente une escale où ils appréhendent - pour la première fois ? - les dimensions matérielles de la civilisation grecque orthodoxe ${ }^{22}$, ce qui explique leurs probables confusions et leurs difficultés à identifier les traits originaux du clergé insulaire.

L'ignorance affichée par ces voyageurs à l'encontre des mœurs grecques autorise des descriptions inédites sur les pratiques religieuses locales. À proximité des Salines, sans doute à l'église grecque Saint-Lazare - bien que celle-ci ne soit jamais expressément nommée -, ils constatent que le service est assuré simultanément selon les deux principaux rites ; Jacques Le Saige observe ainsi que l'office est commun aux Grecs et aux Latins, et il insiste sur la participation à la messe de fidèles qui chantent, les uns en grec, les autres en latin, ce qui suggère une fusion aménagée des rites ${ }^{23}$. Trente ans plus tard, Antoine Regnaut confirme la présence d'un prêtre latin officiant à Saint-Lazare ${ }^{24}$. Si l'on en croit Charles de La Rivière, le clergé attaché à la cathédrale latine de Famagouste, Saint-Nicolas, serait, pour l'essentiel, composé de Grecs qui respecteraient le rite romain durant les offices ${ }^{25}$. Tant de témoignages concordants convainquent de la mixité des rites dans certains lieux fréquentés en commun par les Latins et les Grecs de l'île, attitude peu conforme aux canons religieux, comme le dénonçait Sixte IV dans la bulle qu'il adressait au clergé du royaume, le 24 août $1472^{26}$.

L'attention portée à l'environnement religieux et sacré n'interdit pas des remarques annexes sur le contexte politique, sans constituer une règle générale ; ainsi, Nicolas Loupvant ne prend pas la peine de signaler la présence des Vénitiens sur l'île, alors qu'il ne cesse de se quereller avec le patron de la nave à propos du prix du transport et

19. Anonyme de 1480, p. 107 ; Jacques Le Saige, p. 93.

20. Charles de La Rivière, fol. 84r ; plus généralement : Pierre Barbatre, p. 99-100 ; Jacques Le Saige, p. 138-139.

21. Charles de La Rivière, fol. $84 \mathrm{r}^{\circ}$; Nicolas Loupvant, p. 158.

22. L'anonyme de 1546 , fol. $17 \mathrm{r}^{\circ}$, observe ainsi une simandre, objet dont il semblait ignorer l'existence jusqu'alors.

23. Jacques Le Saige, p. 142.

24. Antoine Regnaut, p. 26.

25. Charles de La Rivière, fol. 83v.

26. Coureas \& Schabel 1997, nº 94. 
des vivres distribuées à bord ; son hostilité à la mentalité mercantile aurait pu susciter des sentiments critiques sur l'administration vénitienne de l'île, mais le bénédictin ne manifeste aucun intérêt quant à la condition des insulaires ${ }^{27}$. L'hégémonie de Venise sur l'île est formulée de manière explicite dans les récits de Sébastien Mamerot, Philippe de Voisins, Charles de La Rivière, Jacques Le Saige et Greffin Affagart, de manière induite dans ceux de l'anonyme de 1480 et de Pierre Barbâtre ${ }^{28}$. L'énoncé de cette situation politique ne provoque aucun commentaire ouvertement défavorable à Venise de la part des voyageurs, sauf sous la plume de Jacques Le Saige qui émet deux critiques fondées sur des plaintes que le voyageur a probablement recueillies de la bouche de marchands : d'une part, Le Saige dénonce la duplicité de Venise qui tire sa suzeraineté sur Chypre du Sultan, auquel elle verse un tribut payé par les impôts prélevés sur la paysannerie; d'autre part, il évoque le monopole exercé par Venise sur les échanges maritimes de l'île ${ }^{29}$.

L'expression de la domination vénitienne sur Chypre se manifeste essentiellement par la présence d'officiers et de soldats. Pierre Barbâtre voit embarquer sur sa galère Ambrogio Contarini qui rentre à Venise après avoir exercé son mandat dans l'île ${ }^{30}$. Jacques Le Saige assure que Venise entretient une garnison insuffisante pour défendre la ville de Famagouste, puisqu'elle ne comprend que 800 soudards ${ }^{31}$. De manière plus exceptionnelle, Georges Lengherand voyage depuis Venise en compagnie de Fiorenza Crispo, mère de Catherine Cornaro, qui rend visite à sa fille à Nicosie, et qui est accueillie aux Salines par le personnel de l'hôtel de la reine ${ }^{32}$. Hormis ces rares occurrences, l'administration civile et militaire de Venise ne paraît guère marquée dans le paysage insulaire ; dans tous les cas, elle suscite moins de curiosité chez nos voyageurs que la présence des églises latines et grecques.

Ce désintérêt pour le statut politique de l'île devenue colonie vénitienne n'interdit pas plusieurs observations passionnantes sur le passé franc et la situation originale de ce royaume, qui conserve des souvenirs matériels de son histoire récente. Ainsi, lors de son passage à Limassol, Denis Possot remarque de belles églises «ruynées du temps passé », "plusieurs beaulx bastimens comme maisons de princes ruynées, à l'une d'icelles sont aux fenestres des armes [...] à quoy nous entendions estre les armes de feu monsieur

27. Nicolas Loupvant, p. 158.

28. Sébastien Mamerot, p. 90 ; Philippe de Voisins, p. 132 ; Charles de La Rivière, fol. $84 \mathrm{r}^{\circ}-\mathrm{v}^{\circ}$; Jacques Le Saige, p. 135 ; Greffin Affagart, p. 243 ; anonyme de 1480, p. 107 ; Pierre Barbatre, p. 99-100.

29. Jacques Le Saige, p. 135.

30. Pierre Barbatre, p. 100, dont le récit est à compléter avec celui, plus précis, de Santo Brasca qui accomplit le même voyage: Grivaud 1990, p. 102 et n. 6.

31. Jacques Le Saige, p. 135.

32. Georges Lengherand, p. 128. 
le duc de Savoye qui fut jadis roy de Cipre » ${ }^{33}$; bien que non corroborées ailleurs ${ }^{34}$, ces informations démontrent une conscience de la présence franque soixante ans après l'éviction de la dynastie des Lusignan ; accessoirement, on peut relever que le souvenir de la venue à Chypre de Louis de Savoie reste vivace, même si le duc n'a jamais réellement exercé son autorité sur le royaume puisque le pouvoir de son épouse, Charlotte de Lusignan héritière légitime de la couronne, fut contesté par son demi-frère, Jacques II le Bâtard ${ }^{35}$. On ne saurait assurer que le même sens de l'observation anime Nicole Le Huen, Sébastien Mamerot et Pierre Le Boucq qui, à plus de soixante ans d'écart, reconnaissent, dans les mêmes termes, sur le mur ouest de l'église des carmes à Nicosie «les armes du Roy de Hierusalem, du Roy de France et dung duc de Normandye » ${ }^{36}$; sans pouvoir l'assurer, ces voyageurs semblent plus s'inspirer du récit de Bernhard von Breytenbach (1483) que soucieux de livrer leurs propres constatations.

La curiosité manifestée à propos des décors héraldiques se trouve complétée par l'attention portée aux inscriptions laissées sur divers monuments. Paphos conserve toujours au milieu d'un paysage de ruines «plusieurs tombes des nobles personnages et leurs épitaphes sont escrit en langage françois $"{ }^{37}$. Plus singulier, Jacques Le Saige et Pierre Le Boucq rapportent une pratique originale des pèlerins de Jérusalem qui, à l'occasion de leur visite à Sainte-Sophie de Nicosie, ou à l'église des franciscains de Limassol, gravent leurs noms sur les portes ou sur les murs des édifices de culte, ce que Camille Enlart avait confirmé à propos des graffitis de Sainte-Sophie de Nicosie et de la chapelle de Pyrga ${ }^{38}$. Parmi les autres références au passé franc de l'île, on constate que Greffin Affagart se plaît à évoquer Jean de Montfort, dont les reliques sont exposées au monastère des franciscains des environs immédiats de Nicosie (Beaulieu), en rappelant qu'il était un comte originaire d'une ville proche de Paris, remarque sans originalité puisqu'elle fut formulée par de nombreux pèlerins avant lui ${ }^{39}$.

\section{Denis Possot, p. 150.}

34. On ne trouve aucune mention d'armoiries franques sur des bâtiments civils de Limassol dans les travaux de Enlart 1899, vol. II, ou dans le volume dirigé par de Vaivre, Plagnieux 2006.

35. On rappellera que Louis de Savoie épousa Charlotte de Lusignan et fut couronné roi de Chypre le 7 octobre 1459 : Mas Latrie 1882, p. 390 ; Georgios Boustronios, p. 52 et 364 ; Florio Bustron, p. 392 ; Hill 1948, vol. III, p. 549.

36. Pierre Le Boucq, fol. $32 \mathrm{v}^{\circ}$, qui s'inscrit dans la filiation ouverte par Nicole Le Huen : « Nostre maison des carmes est pres du palay du roy, et fust fondée par les seigneurs de France, car on en voit lapparence en lesglise par les armes du roy de Hierusalem, du roy de France, du duc de Normandie », fol. n. n. ; et Sébastien Mamerot, p. 90.

37. Anonyme de 1546 , fol. $18 \mathrm{r}^{\circ}$.

38. Jacques Le Saige, p. 138 ; Pierre Le Boucq, fol. $32 \mathrm{v}^{\circ}:$ « la avons nous trouve plusieurs noms de pelerins venus de la saincte cité, natifs de Vallenciennes»; sur les graffitis de Sainte-Sophie : Enlart 1899, vol. I, p. 132-133; sur ceux de la chapelle de Pyrga : ibid., vol. II, p. 436-439, et l'étude de Schryver, Schabel 2007, p. 327-334.

39. Greffin Affagart, p. 244 ; Jacques Le Saige, p. 139. De nombreux autres voyageurs rapportent 
C'est à propos de Paphos que les observations rapportées par les voyageurs français prennent une tournure originale car, lorsqu'ils sont conduits dans les hypogées connus sous le nom de Grotte des sept dormants, leurs interprétations n'associent pas le site à une période de l'Antiquité mais à des légendes médiévales. Ainsi, à la fin de l'été 1490, Jean de Cucharmoys et Philippe de Voisins distinguent, au milieu des hypogées, une chapelle qui abrite la tombe de Raymondin «sieur de Lezinam », mari de Mélusine et père de Geoffroy la Grande Dent, qui devint roi de Chypre et fit apporter le corps de son père : "Et de ceste generation sont descendus les rois de Chippre, jusques a present qu'ilz sont ausdicts Venitiens, qui leur est chose griefve et seroient volountiers ez mains des François, car ilz en ayment naturellement la nation ». Ce rappel de la généalogie mythique des Lusignan fait écho au roman de la légende de Mélusine ${ }^{40}$, dont nos voyageurs eurent probablement connaissance par les romans de Jean d'Arras (1392-1393) ou de Coudrette (début du Xv ${ }^{\text {e }}$ siècle) ${ }^{41}$; il semble en effet exclu que cette légende puisse s'appuyer sur une tradition circulant à Chypre, compte tenu de la rareté des références au mythe fondateur de la dynastie poitevine dans la littérature franque locale ${ }^{42}$. L'aspect exceptionnel de cette remarque, confirmée par le silence des voyageurs des $\mathrm{XV}^{\mathrm{e}}$ et $\mathrm{XVI}^{\mathrm{e}}$ siècles - de culture française ou étrangère -, laisse supposer que la référence appartient à une vulgate colportée par un membre de la compagnie pèlerine ; l'anonyme qui se rend à la Grotte des sept dormants, en 1546, tait, en effet, toute référence à la tombe de Raymondin, aux Lusignan et aux Francs ${ }^{43}$.

L'expression du regret que ressentiraient les Francs d'être passés sous la sujétion de Venise est très rare, appartenant à deux observateurs seulement : d'abord, à Philippe de Voisins, seul voyageur de la fin $d u x^{e}$ siècle transmettant un avis de noble ou de bourgeois franc mécontent de la nouvelle administration vénitienne ${ }^{44}$. Dix-sept ans plus tard, Charles de La Rivière répète une critique du même ordre contre Venise, au début du mois de septembre 1507, soit plus de trente années après l'installation du pouvoir vénitien sur l'île :

«Ladicte cite [Nicosie] et toult le pays est subject a la Seigneurie de Venise depuys dix huyt ou xx ans en ca depuys le quel tempz, ilz ont change toult lordre et maniere de faire a coustume. Car au paravant ilz faisoient les procez et [...] et plaidoient en franchoys.

aveuglément la même information, $c f$. Trélat 2009, vol. II, p. 214-222 ; en réalité, Jean de Montfort était un chevalier de Languedoc arrivé à Chypre avec la croisade de Louis IX en 1248-1249, décédé durant l'hivernage de l'expédition à Chypre ; sur le culte de saint Jean de Montfort à Beaulieu : Grivaud 2003, p. 73-74.

40. Jean de Cucharmois, p. 130 ; Philippe de Voisins, p. 131-132.

41. Jean d'Arras, 1932 ; Coudrette 1982.

42. Les mentions de Mélusine dans la culture de cour à Chypre sont peu explicites : on peut douter de l'identification proposée par Enlart 1899, vol. I, p. 175 ; une référence indirecte est signalée par Richard 1952, p. 109 n. 1, référence davantage développée par Richard 1993, p. 251-252.

43. Anonyme de 1546 , fol. $18 r^{\circ}$.

44. Philippe de Voisins, p. 132. 
Et maintenant ilz les font en italien de quoy les habitans sont bien mal contantz. Car tous ceulx du pays et speciallement les gentils hommes sont aussi bons franchoys que nous sommes en France. Et sont mervilleusement mal contentz destre en la subiection de Venise. ${ }^{45}$

La nature des griefs transmis par de La Rivière laisse comprendre que le pèlerin a échangé directement avec des membres de familles nobles franques, les seuls capables de déplorer la transformation des procédures judiciaires ; en effet, celles-ci se déroulent désormais en italien, ce qui entrave l'application de la coutume franque, où les procès se tenaient en français, selon les normes fixées par le Livre de Jean d'Ibelin depuis $1369{ }^{46}$. Dès 1474, Venise s'était engagée à respecter les codes de lois féodales du royaume franc, mais on sait qu'il faut attendre la volonté réformatrice du doge Andrea Gritti pour qu'une traduction italienne des Assises soit entreprise, en 1521, et publiée en $1525^{47}$. La plainte répercutée par Charles de La Rivière constitue donc un témoignage précieux en ce qu'il dévoile combien la phase de transition institutionnelle a longtemps soulevé des tensions entre Venise et ses feudataires chypriotes. L'aspect linguistique du problème laisse comprendre que la pratique du français revêt au début du $\mathrm{XvI}^{\mathrm{e}}$ siècle un caractère identitaire marqué. Il convient de souligner qu'aux oreilles de Charles de La Rivière, la langue pratiquée par les Francs de Chypre permet l'inter-compréhension, au même titre que n'importe quelle autre variante des parlers de la France de la Renaissance.

De ces remarques, il ressort qu'on ne saurait relever une spécificité dans le regard que les voyageurs de langue française portent sur Chypre, après 1474, par rapport aux voyageurs issus d'autres nations occidentales. Aucun des récits étudiés n'évoque un lien privilégié entre l'île et le royaume des Valois. L'ignorance du passé de l'île est tempérée par une certaine sensibilité aux vestiges matériels laissés par la présence franque, mais seul Charles de La Rivière se départit de l'indifférence habituelle pour prendre la défense de ces nobles qui parlent la même langue que lui. Sinon, on demeure frappé de constater à quel point les voyageurs reproduisent une vulgate qui attribue la ruine de Limassol à un roi d'Angleterre... L'absence de références au passé mythologique de l'île atteste encore la faible diffusion des connaissances élaborées par les humanistes de la Renaissance italienne ${ }^{48}$. Assurément, le groupe de textes pris en examen convainc que le but des voyageurs - accomplir le pèlerinage de Terre sainte - ne les prédisposait guère à saisir les caractères culturels originaux d'un royaume franc passé sous domination vénitienne. Chypre constituait une étape dont on percevait l'identité religieuse, à défaut d'identifier

45. Charles de La Rivière, fol. $84 \mathrm{r}^{\circ}-\mathrm{v}^{\circ}$.

46. Voir la récente édition de référence : Jean d'Ibelin, 2003.

47. Grivaud 2009, p. 240-245.

48. Grivaud 2009, p. 219-223 ; on peut mesurer le retard pris par ces voyageurs dans la connaissance du passé antique de Chypre en confrontant leurs récits avec les traités des lettrés italiens tels qu'ils viennent d'être analysés par Calvelli 2009. 
des références « nationales », qui n'avaient guère de sens dans les constructions idéologiques de l'époque.

Département d'histoire, Université de Rouen, Normandie Université

\section{BIBLIOGRAPHIE}

\section{Récits de voyage (l'année du passage à Chypre entre parenthèses)}

ANONYMe (1480): Le voyage de la saincte cyté de Hierusalem avec la description des lieux, portz, villes, citez et aultres passaiges fait en l'en mil quatre cents quatre vingtz, éd. Ch. Schefer, Paris, 1882 [cité dans l'éd. Grivaud 1990, p. 106-111].

Anonyme (1546) : Pèlerinage de Douai à Jérusalem, BNF, ms fr. 13083.

Antoine Regnault (1549) : Discours du voyage d'Outremer au Sainct Sepulcre de Jerusalem, et autres lieux de la Terre Saincte, Lyon, 1573.

BARTHÉlémy dE SALIGNAC (avant 1525) : Itinerarii Terre sancte inibique sacrorum locorum ac rerum clarissima descriptio omnibus Sacre Scripture tractatoribus utilissima..., Lyon, 1525.

Charles de LA Rivière (1507) : Le voyage en Terre sainte fait en 1507 par Charles de La Rivière, prebstre, chanoine de Lisieux, qui a escrit de sa main le contenu de ce livre, qui est une relation de son voyage, Rouen, Bibliothèque Municipale, ms. 1118 (U 100) [récit presque identique à celui de Pierre Mésenge déposé à la Bibliothèque Municipale d'Amiens, fonds Lescalopier 5215].

Denis Possot (1532) : Le voyage de Terre Sainte composé par maître Denis Possot et achevé par messire Charles Philippe, 1532, éd. Ch. Schefer, Paris, 1890.

Georges Lengherand (1486) : Voyage de Georges Lengherand, mayeur de Mons en Hainaut, à Venise, Rome, Jérusalem, Mont Sinaï et de Kayre 1485-1486, éd. G. Ménilgalise, Mons, 1861 [cité dans l'éd. Grivaud 1990, p. 127-129].
GrefFin Affagart $(1519,1533-1534)$ : Relation de Terre sainte (1533-1534), éd. J. Chavagnon, Paris, 1902.

Jacques Le SAIGe (1518) : Voyage de Jacques Le Saige, de Douai à Rome, Notre-Dame de Lorette, Venise, Jérusalem et autres saints lieux, éd. R.-H. Duthilloeul, Douai, 1851.

JEAn DE CuCharmoys (1490): Le sainct voyage de Hierusalem ou petit traicté du voyage de Hierusalem de Rome et de Sainct-Nicolas de Bar en Pouille, éd. A. de Masry, Genève, 1889.

Nicole Le Huen (1479 ?), Des Sainctes peregrinations de Iherusalem, Lyon, 1488.

Nicolas Loupvant (1531) : Dom Loupvent, Récit d'un voyageur lorrain en Terre Sainte au $\mathrm{XVI}^{e}$ siècle, éd. J. Lanher et Ph. Martin, Nancy et Bar-le-Duc, 2007.

PhilipPe DE VoIsins (1490) : Voyage à Jérusalem de Philippe de Voisins seigneur de Montaut, éd. Ph. Tamizey de Larroque, Paris, 1883 [cité dans l'éd. Grivaud 1990, p. 131-132].

Pierre Barbatre (1480) : « Le voyage de Pierre Barbatre à Jérusalem en 1480 », éd. P. TuccoChala et N. Pinzuti, Annuaire-Bulletin de la Société de l'histoire de France (1972-1973), p. 73-172 [cité dans l'éd. Grivaud 1990, p. 98-101].

Pierre Le BoucQ (1548) : Le sainct voyage de Jérusalem, Valenciennes, Bibliothèque Municipale, $m s .489$.

Sébastien Mamerot (c. 1480) : Les Passages d'Oultremer, Paris, 1518 [cité dans l'éd. Grivaud 1990, p. 89-91]. 


\section{Autres sources et études}

Baglioni (D.), 2006, La scripta italoromanza del Regno di Cipro, Rome.

BeiHammer (A.), 2007, Griechische Briefe und Urkunden aus dem Zypern der Kreuzfahrerzeit. Die Formularsammlung eines königlichen Sekretärs im Vaticanus Palatinus Graecus 367, Nicosie.

Calvelli (L.), 2009, Cipro e la memoria dell'Antico fra Medioevo e Rinascimento. La percezione del passato romano dell'isola nel mondo occidentale, Venise.

Chateaubriand (F.-R. de), 1836, « Itinéraire de Paris à Jérusalem », dans CEuvres complètes, vol. X/2, Paris.

Coudrette, 1982, Le Roman de Mélusine ou Histoire de Lusignan par Coudrette, éd. E. Roach, Paris.

Coureas (N.), Schabel (Chr.) éds, 1997, The Cartulary of the Cathedral of the Holy Wisdom of Nicosia, Nicosie.

ENLART (C.), 1899, L'art gothique et la Renaissance en Chypre, Paris, 2 vol.

Florio Bustron, Chronique de l'île de Chypre, éd. R. de Mas Latrie, Paris, 1886 [rééd. sous le titre Historia overo commentarii di Cipro, Nicosie, 1998].

Georgios Boustronios, $\Delta \iota \dot{\gamma} \gamma \eta \sigma \varsigma$ Koovixas Kv́л@ov, éd. G. Kechagioglou, Nicosie, 1997.

Grivaud (G.), 1990, Excerpta Cypria Nova. Voyageurs occidentaux à Chypre au XV siècle, Nicosie.

Grivaud (G.), 1992, «Ordine della Secreta di Cipro. Florio Bustron et les institutions franco-byzantines afférentes au régime agraire

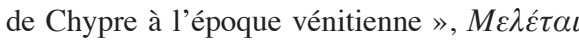

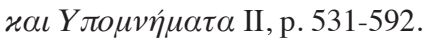

GrivaUd (G.), 2001, «Une petite chronique chypriote du $\mathrm{XV}^{\mathrm{e}}$ siècle », dans M. Balard, B. Kezdar, J. Riley-Smith (éd.), Dei Gesta per Francos. Études sur les croisades dédiées à Jean Richard, Aldershot, p. 317-338.
Grivaud (G.), 2003, « Pèlerinages grecs et pèlerinages latins dans le royaume de Chypre (1192-1474). Concurrence ou complémentarité ? », dans C. Vincent (éd.), Identités pèlerines, Mont Saint-Aignan, p. 67-76.

Grivaud (G.), 2009, Entrelacs chiprois. Essai sur les lettres et la vie intellectuelle dans le royaume de Chypre (1191-1570), Nicosie.

Hill (G.), 1948, A History of Cyprus, Cambridge, vol. II-III.

Iorga (N.), 1931, France de Chypre, Paris [rééd. Paris, 1966].

JEAn D'Arras, Mélusine, Roman du XIVe siècle, éd. L. Stouff, Dijon, 1932 [trad. M. Perret, Le Roman de Mélusine ou l'histoire de Lusignan, Paris, 1979].

JEAN D'Ibelin, Le Livre des Assises, éd. P.W. Edbury, Leyde-Boston, 2003.

Marcellus (Comte de), 1839, Souvenirs de l'Orient, Paris, 2 vol.

Mas Latrie (L. de), 1879, L'île de Chypre, sa situation présente et ses souvenirs du Moyen Âge, Paris.

Mas Latrie (L. de), 1882, « Documents nouveaux servant de preuves à l'histoire de l'île de Chypre sous le règne des princes de la maison de Lusignan », Collection des documents inédits : Mélanges historiques IV, p. 337-619.

Michaud (J.), Poujoulat (J. J.), 1833-1835, Correspondance d'Orient, 1830-1831, Paris, 7 vol.

Nicolaou-Konnari (A.), 1995, « H $\gamma \lambda \omega ́ \sigma \sigma \alpha$

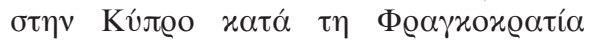

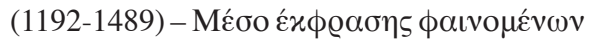

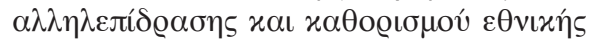

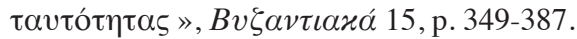

RichARD (J.), 1952, « La révolution de 1369 dans le royaume de Chypre », Bibliothèque de l'École des Chartes CX, p. 108-123 [repr. 
dans J. Richard, Orient et Occident au Moyen $\hat{A} g e$. Contacts et relations XII $-\mathrm{XIV}{ }^{e}$ siècles, Londres, 1976, étude ${ }^{\circ}$ XVI].

RichaRd (J.), 1962, Documents chypriotes des archives du Vatican (XIVe et XVe siècles), Paris.

RichaRd (J.), 1983, Le Livre des Remembrances de la Secrète du Royaume de Chypre (14681469), Nicosie.

RichARD (J.), 1986, « La diplomatique royale dans le Royaume d'Arménie et de Chypre ( $\mathrm{XII}^{\mathrm{e}}-\mathrm{XV}^{\mathrm{e}}$ siècles) », Bibliothèque de l'École des Chartes 144, p. 69-86 [repr. dans J. Richard, Croisades et États latins d'Orient, Londres, 1992, étude $n^{\circ}$ XIX].

Richard (J.), 1987, « Culture franque et culture grecque : le royaume de Chypre au $\mathrm{XV}^{\mathrm{e}}$ siècle ", Byzantinische Forschungen XI, p. 399-415 [repr. dans J. Richard, Croisades et États latins d'Orient, Londres, 1992, étude $\mathrm{n}^{\circ} \mathrm{XVIII]}$.

Richard (J.), 1993, « Des Lusignan mythiques au mythe des Lusignan : un "petit Lusignan" au $\mathrm{XV}^{\mathrm{e}}$ siècle ", dans C. Mutifian, Les
Lusignans et L'Outre Mer, Poitiers-Lusignan, p. 251-259.

RichARD (J.), 2003, « Le plurilinguisme dans les actes de l'Orient latin », La langue des actes. Actes du XI ${ }^{e}$ congrès international de diplomatique (Troyes 11-13 septembre 2003), étude en ligne http://elec.enc.sorbonne.fr/ CID2003.

Schryver (J.G.), Schabel (Chr.), 2003, « The graffiti in the "Royal Chapel" of Pyrga, RDAC, p. 327-334.

Trélat (Ph.), 2009, Nicosie, une capitale de l'Orient latin, société, économie et espace urbain (1192-1474), thèse Université de Rouen, 2 vol.

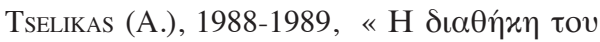

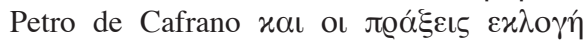

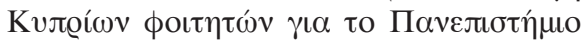

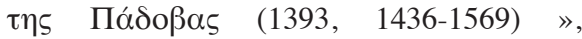

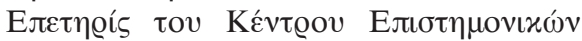
E@evvóv XVII, p. 261-292.

Vaivre (J.-B. de), Plagnieux (Ph.), éds, 2006, L'art gothique en Chypre, Paris. 